\title{
A SPACE OF PROJECTIONS ON THE BERGMAN SPACE
}

\section{Oscar Blasco and Salvador Pérez-Esteva}

Universidad de Valencia, Departamento de Matemáticas 46100-Burjassot (Valencia), Spain; Oscar.Blasco@uv.es

Universidad Nacional Autónoma de México, Instituto de Matemáticas Unidad Cuernavaca A.P. 273-3 ADMON 3, Cuernavaca, Mor., 62251, México; salvador@matcuer.unam.mx

\begin{abstract}
We define a set of projections on the Bergman space $A^{2}$, which is parameterized by an affine subset of a Banach space of holomorphic functions in the disk and which includes the classical Forelli-Rudin projections.
\end{abstract}

\section{Introduction}

Recall that the Bergman projection of $L^{2}(\mathbf{D})$ onto the holomorphic Bergman space $A^{2}=L^{2}(\mathbf{D}) \cap \mathscr{H}(\mathbf{D})$, where $\mathscr{H}(\mathbf{D})$ denotes the space of holomorphic functions in the unit disk, is given by

$$
P \varphi(z)=\int_{\mathbf{D}} \frac{\varphi(w)}{(1-z \bar{w})^{2}} d A(w)
$$

where $d A$ is the normalized Lebesgue measure in the disk. Recall also the family of Forelli-Rudin projections parameterized by $\alpha>-1$

$$
P_{\alpha} \varphi(z)=\int_{\mathbf{D}}(\alpha+1)\left(\frac{1-|w|^{2}}{1-z \bar{w}}\right)^{\alpha} \frac{\varphi(w)}{(1-z \bar{w})^{2}} d A(w) .
$$

These are the orthogonal projections of the weighted $L^{2}\left(\mathbf{D},(1-|w|)^{\alpha} d A(w)\right)$ onto $\mathscr{H}(\mathbf{D}) \cap L^{2}\left(\mathbf{D},(1-|w|)^{\alpha} d A(w)\right)$. It is well known (see [6, Th. 7.1.4]) that $P_{\alpha}$ is a continuous projection of $L^{2}(\mathbf{D})$ onto $A^{2}$, for each $\alpha>-1 / 2$.

Since

$$
\left\{\frac{1-|w|^{2}}{1-z \bar{w}}, z, w \in \mathbf{D}\right\} \subset \mathbf{D}_{1}
$$

where $\mathbf{D}_{1}=\{z:|z-1|<1\}$, we may replace the function $g_{\alpha}(\zeta)=(\alpha+1) \zeta^{\alpha}$ in the definition of $P_{\alpha}$ by any holomorphic function $g$ on $\mathbf{D}_{1}$ to obtain an operator $T_{g}$ mapping the space $C_{c}(\mathbf{D})$ of compactly supported continuous functions defined on D into $A^{2}$. An equivalent formulation of the operators defined this way was given by Bonet, Engliš and Taskinen in [1] to construct continuous projections in weighted $L^{\infty}$ spaces of $\mathbf{D}$ into $\mathscr{H}(\mathbf{D})$. The purpose of this paper is to study the space $\mathscr{P}$ of all holomorphic functions $g \in \mathbf{D}_{1}$, for which the corresponding operator $T_{g}$ can be extended continuously to $L^{2}(\mathbf{D})$. In particular we study the set $\mathscr{P}_{0}$ of those functions $g \in \mathscr{P}$ that define continuous projections on $A^{2}$. For notational convenience we will translate the functions in $\mathscr{P}$ to the unit disk $\mathbf{D}$.

doi:10.5186/aasfm.2010.3512

2000 Mathematics Subject Classification: Primary 46E20.

Key words: Bergman spaces, projection.

First author partially supported by the spanish grant MTM2008-04594/MTM.

Second author partially supported by Conacyt-DAIC U48633-F. 
We will prove that $\mathscr{P}$ is a Banach space when we define the norm of $g \in \mathscr{P}$ as the operator norm of the operator $T_{g}$ and that $\Phi(g)=\int_{0}^{1} g(r) d r$ defines a bounded linear functional in $\mathscr{P}^{*}$. We give an analytic description of the elements of $\mathscr{P}$ and show that if $g \in \mathscr{P}$ then either $T_{g}$ is identically zero on $A^{2}$ or it is a multiple of a continuous projection onto $A^{2}$, implying that $\mathscr{P}_{0}=\Phi^{-1}(\{1\})$ is a closed affine subspace of $\mathscr{P}$.

As usual, for each $z \in \mathbf{D}, \phi_{z}$ will denote by $\phi_{z}$ the Möbius transform $\phi_{z}(w)=\frac{z-w}{1-\bar{z} w}$ which satisfies $\left(\phi_{z}\right)^{-1}=\phi_{z}$ and $\phi_{z}^{\prime}(w)=-\frac{1-|z|^{2}}{(1-\bar{z} w)^{2}}$. Throughout this paper we will write

$$
\psi_{z}(w)=\frac{1-|w|^{2}}{1-z \bar{w}}
$$

and

$$
\mathbf{H}=\{z \in \mathbf{C}: \operatorname{Re}(z)>1 / 2\} .
$$

Clearly the mapping $z \rightarrow \frac{1}{1-z}$ is a bijection of $\mathbf{D}$ onto $\mathbf{H}$, and

$$
\psi_{z}(w)=1-\bar{w} \phi_{w}(z) .
$$

\section{A space of projections on $A^{2}$}

Let us start by presenting our new definitions and spaces of projections.

Definition 1. Let $g$ be holomorphic in $\mathbf{D}$. We define

$$
T_{g} \varphi(z)=\int_{\mathbf{D}} g\left(\bar{w} \phi_{w}(z)\right) \varphi(w) \frac{d A(w)}{(1-z \bar{w})^{2}}
$$

for any $\varphi \in C_{c}(\mathbf{D})$. We denote by $\mathscr{P}$ (resp. $\left.\mathscr{P}_{0}\right)$ the space of holomorphic functions $g \in \mathscr{H}(\mathbf{D})$ such that $T_{g}$ extends continuously to $L^{2}(\mathbf{D})$ (resp. $T_{g}$ is a projection on the Bergman space $A^{2}$ ). We provide the space $\mathscr{P}$ with the norm $\|g\|_{\mathscr{P}}=$ $\left\|T_{g}\right\|_{L^{2}(\mathbf{D}) \rightarrow L^{2}(\mathbf{D})}$.

Remark 2. In [1] it was introduced, for each $F$ holomorphic in $\mathbf{H}$ the operator

$$
S_{F} \varphi(z)=\int_{D} F\left(\frac{1-z \bar{w}}{1-|w|^{2}}\right) \varphi(w) \frac{d A(w)}{\left(1-|w|^{2}\right)^{2}} .
$$

We have $T_{g}=S_{F}$, with $F(\eta)=\frac{1}{\eta^{2}} g\left(1-\frac{1}{\eta}\right)$. We will say that such $F \in \mathscr{P}\left(\right.$ resp. $\left.\mathscr{P}_{0}\right)$ if $g \in \mathscr{P}$ (resp. $\left.\mathscr{P}_{0}\right)$.

Example 3. Let $g_{\alpha}(z)=(\alpha+1)(1-z)^{\alpha}$ for every $\alpha>-1$. Then $g_{\alpha} \in \mathscr{P}_{0}$ for $\alpha>-1 / 2$. In fact by (1) we have $T_{g_{\alpha}}=P_{\alpha}$, which is a bounded projection from $L^{2}(\mathbf{D})$ into $A^{2}$ if and only if $\alpha>-1 / 2$.

Example 4. If $P(z)=\sum_{k=0}^{N} a_{k} z^{k}$ is a polynomial then $P \in \mathscr{P}$. Moreover, $P \in \mathscr{P}_{0}$ if and only if $\sum_{k=0}^{N} \frac{a_{k}}{(k+1)}=\int_{0}^{1} P(r) d r=1$.

Proof. Write $P(z)=\sum_{k=0}^{N} b_{k}(1-z)^{k}$ where $b_{k}=(-1)^{k} \frac{P^{(k)}(1)}{k !}$. Hence

$$
T_{P}=\sum_{k=0}^{N} \frac{b_{k}}{(k+1)} P_{k} .
$$


This shows that $T_{P} \in \mathscr{P}$ and $\|P\|_{\mathscr{P}} \leq \sum_{k=0}^{N} \frac{\left|b_{k}\right|}{(k+1)}\left\|P_{k}\right\|$. On the other hand $T_{P} \in \mathscr{P}_{0}$ if and only if $\sum_{k=0}^{N} \frac{b_{k}}{(k+1)}=1$. Notice now that $\sum_{k=0}^{N} \frac{b_{k}}{(k+1)}=\int_{0}^{1} P(r) d r$ to conclude the proof.

Example 5. If $g \in \mathscr{H}(\mathbf{D})$ is such that $(1-z)^{-\alpha} g(z)$ is bounded for some $\alpha>-1 / 2$ then $g \in \mathscr{P}$ and $\|g\|_{\mathscr{P}} \leq C \sup _{|z|<1}\left|(1-z)^{-\alpha} g(z)\right|$. In particular the space of bounded holomorphic functions $H^{\infty}(\mathbf{D})$ is contained in $\mathscr{P}$ and $\|f\|_{\mathscr{P}} \leq C\left\|_{f}\right\|_{\infty}$.

Proof. Use the fact that $P_{\alpha}^{*} \varphi(z)=\int_{D} \frac{\left(1-|w|^{2}\right)^{\alpha}}{|1-\bar{w} z|^{2+\alpha}} \varphi(w) d A(w)$ also defines a bounded operator on $L^{2}(\mathbf{D})$ (see [5, Theorem 1.9]).

Proposition 6. Let $g:\{z:|z-1|<2\} \rightarrow \mathbf{C}$ be holomorphic such that $g(z)=$ $\sum_{n=1}^{\infty} a_{n}(1-z)^{n}$ for $|z-1|<2$. If $\sum_{n=0}^{\infty} \frac{2^{n}\left|a_{n}\right|}{(n+1)^{5 / 4}}<\infty$, then $g \in \mathscr{P}$ and

$$
\|g\|_{\mathscr{P}} \leq C \sum_{n=0}^{\infty} \frac{2^{n}\left|a_{n}\right|}{(n+1)^{5 / 4}} .
$$

Moreover, $g \in \mathscr{P}_{0}$ if and only if $\sum_{n=0}^{\infty} \frac{a_{n}}{n+1}=1$.

Proof. Indeed, the norm $\left\|P_{n}\right\|=\frac{\sqrt{(2 n) !}}{n !}($ see $[2,3])$. Then for $\varphi \in C_{c}(\mathbf{D})$

$$
T_{g} \varphi(z)=\sum_{n=1}^{\infty} \frac{a_{n}}{(n+1)} P_{n} \varphi(z)
$$

and

$$
\|g\|_{\mathscr{P}} \leq \sum_{n=0}^{\infty} \frac{\left|a_{n}\right| \sqrt{(2 n) !}}{(n+1) n !} .
$$

Finally observe that, from Stirling's formula, $\frac{\sqrt{(2 n) !}}{(n+1) n !} \sim \frac{2^{n}}{(n+1)^{5 / 4}}$. To conclude the result note that $\sum_{n=0}^{\infty} \frac{\left|a_{n}\right|}{n+1}<\infty$ and

$$
T_{g} \varphi(z)=\left(\sum_{n=1}^{\infty} \frac{a_{n}}{(n+1)}\right) \varphi(z),
$$

for $\varphi \in A^{2}$.

Example 7. Let $h_{\beta}(z)=A_{\beta}(1+z)^{-\beta}$ for $\beta>0$ where $A_{\beta}=\frac{1-\beta}{2^{-\beta+1}-1}$ if $\beta \neq 1$ and $A_{1}=(\log 2)^{-1}$. Then $h_{\beta} \in \mathscr{P}_{0}$ for $0<\beta<5 / 4$. have

Proof. Since $\frac{1}{(1-w)^{\beta}}=\sum_{n=0}^{\infty} \beta_{n} w^{n}$ for $\beta>0,|w|<1$, where $\beta_{n} \sim(n+1)^{\beta-1}$, we

$$
h_{\beta}(z)=\frac{A_{\beta}}{2^{\beta}(1-(1-z) / 2)^{\beta}}=\sum_{n=0}^{\infty} A_{\beta} 2^{-(n+\beta)} \beta_{n}(1-z)^{n} .
$$

Now Proposition 6 implies $h_{\beta} \in \mathscr{P}$.

Note that

$$
1=\int_{1}^{2} A_{\beta} s^{-\beta} d s=\int_{0}^{1} h_{\beta}(r) d r=\sum_{n=0}^{\infty} \frac{A_{\beta} 2^{-(n+1)} \beta_{n}}{n+1} .
$$

Apply again Proposition 6 to finish the proof. 
Let us now give some necessary conditions that functions $g$ in $\mathscr{P}$ should satisfy.

Theorem 8. If $g \in \mathscr{P}$, then

$$
\begin{array}{r}
\sup _{z \in \mathbf{D}}\left\{\int_{\mathbf{D}}\left|g\left(\bar{w} \phi_{w}(z)\right)\right|^{2} d A(w)\right\}^{1 / 2} \leq 2\|g\|_{\mathscr{P}}, \\
\left(\int_{0}^{1}|g(r)|^{2} d r\right)^{1 / 2} \leq 2\|g\|_{\mathscr{P}}, \\
\left(\int_{0}^{1}\left(\int_{\mathbf{D}} \frac{\mid g(r u))\left.\right|^{2}}{|1-r u|^{4}} d A(u)\right)\left(1-r^{2}\right)^{2} r d r\right)^{1 / 2} \leq 2\|g\|_{\mathscr{P}} .
\end{array}
$$

Proof. If $g \in \mathscr{P}$ and $\varphi \in C_{c}(\mathbf{D})$ one has $T_{g} \varphi \in A^{2}$. Hence for each $z \in \mathbf{D}$

$$
\left|T_{g} \varphi(z)\right| \leq \frac{\left\|T_{g} \varphi\right\|_{2}}{(1-|z|)} \leq \frac{\|g\|_{\mathscr{P}}\|\varphi\|_{2}}{(1-|z|)} .
$$

Therefore

$$
\left|\int_{\mathbf{D}} g\left(\bar{w} \phi_{w}(z)\right) \varphi(w) \frac{d A(w)}{(1-z \bar{w})^{2}}\right| \leq \frac{\|g\|_{\mathscr{P}}\|\varphi\|_{2}}{(1-|z|)}
$$

Then by duality,

$$
\left\{\int_{\mathbf{D}}\left|g\left(\bar{w} \phi_{w}(z)\right)\right|^{2} \frac{d A(w)}{|1-z \bar{w}|^{4}}\right\}^{1 / 2} \leq \frac{\|g\|_{\mathscr{P}}}{(1-|z|)} \leq 2 \frac{\|g\|_{\mathscr{P}}}{\left(1-|z|^{2}\right)} .
$$

Let us show the following formula:

$$
\overline{\phi_{z}(u)} \phi_{\phi_{z}(u)}(z)=u \overline{\phi_{u}(z)}
$$

Indeed, since

$$
1-\left|\phi_{z}(u)\right|^{2}=\frac{\left(1-|z|^{2}\right)\left(1-|u|^{2}\right)}{|1-\bar{z} u|^{2}}
$$

then

$$
\psi_{z}\left(\phi_{z}(u)\right)=\frac{1-\left|\phi_{z}(u)\right|^{2}}{1-\overline{\phi_{z}(u)} z}=\frac{\left(1-|u|^{2}\right)}{(1-\bar{z} u)}=\overline{\psi_{z}(u)} .
$$

Now (6) follows from (1) and (7)

$$
\overline{\phi_{z}(u)} \phi_{\phi_{z}(u)}(z)=1-\psi_{z}\left(\phi_{z}(u)\right)=u \overline{\phi_{u}(z)} .
$$

Changing the variable $u=\phi_{z}(w)$ in (5) and using (6) we obtain

$$
\left\{\int_{\mathbf{D}}\left|g\left(u \overline{\phi_{u}(z)}\right)\right|^{2} d A(u)\right\}^{1 / 2} \leq 2\|f\|_{\mathscr{P}} .
$$

Now replacing $u$ and $\bar{z}$ by $\bar{w}$ and $z$ respectively the inequality (2) is achieved.

Part (3) follows selecting $z=0$ in (2). 
Part (4) follows from (2) replacing the supremum by an integral over $\mathbf{D}$ and changing the variable $u=\phi_{w}(z)$,

$$
\begin{aligned}
\int_{\mathbf{D}} \int_{\mathbf{D}}\left|g\left(\bar{w} \phi_{w}(z)\right)\right|^{2} d A(w) d A(z) & =\int_{\mathbf{D}}\left(\int_{\mathbf{D}} \frac{\mid g(\bar{w} u))\left.\right|^{2}}{|1-\bar{w} u|^{4}} d A(u)\right)\left(1-|w|^{2}\right)^{2} d A(w) \\
& =\int_{\mathbf{D}}\left(\int_{\mathbf{D}} \frac{\mid g(|w| u))\left.\right|^{2}}{|1-| w|u|^{4}} d A(u)\right)\left(1-|w|^{2}\right)^{2} d A(w) \\
& =\int_{0}^{1}\left(\int_{\mathbf{D}} \frac{|g(r u)|^{2}}{|1-r u|^{4}} d A(u)\right)\left(1-r^{2}\right)^{2} r d r
\end{aligned}
$$

Remark 9. $\left(\mathscr{P},\|\cdot\|_{\mathscr{P}}\right)$ is a normed space and $\Phi(g)=\int_{0}^{1} g(r) d r \in \mathscr{P}^{*}$. Indeed, the only condition which needs a proof is the fact that $\|g\|_{\mathscr{P}}=0$ implies $g=0$. It follows from (3) that if $\|g\|_{\mathscr{P}}=0$, then $g(r)=0$ for $0<r<1$. Hence by analytic continuation, $g(z)=0$ for $z \in \mathbf{D}$. Notice also that (3) implies $\|\Phi\| \leq 2$.

Remark 10. The space $\mathscr{P}$ is not invariant under under rotations. Given $\theta \in$ $[0,2 \pi)$ denote $R_{\theta}(f)(z)=f\left(e^{i \theta} z\right)$ for $f \in \mathscr{H}(\mathbf{D})$. Observe that $R_{\theta} T_{g}(\varphi)=T_{g}\left(R_{\theta} \varphi\right)$. However, " $T_{g}$ is bounded in $L^{2}(\mathbf{D})$ does not imply $T_{R_{\theta} g}$ is bounded in $L^{2}(\mathbf{D})$ ". For instance, the function $g(z)=(1+z)^{-1 / 2}$ belongs to $\mathscr{P}$, but by (3), its reflection $g(z)=(1-z)^{-1 / 2} \notin \mathscr{P}$.

Let us now also give some necessary conditions to belong to the class $\mathscr{P}_{0}$.

Theorem 11. If $g \in \mathscr{P}_{0}$ then

$$
\int_{\mathbf{D}} g\left(u \overline{\phi_{u}(z)}\right) \psi(u) d A(u)=\psi(0)
$$

for all $\psi \in A_{2}$ and $z \in \mathbf{D}$. In particular,

(i) If $g \in \mathscr{P}_{0}$ then $\int_{0}^{1} g(r) d r=1$.

(ii) Let $S_{2}=\left\{\bar{z}\left(1-|z|^{2}\right) \varphi(\bar{z}): \varphi \in A^{2}\right\}$. If $g \in \mathscr{P}_{0}$ and $g^{\prime} \in \mathscr{P}$ then $S_{2} \subset$ $\operatorname{Ker}\left(T_{g^{\prime}}\right)$.

Proof. Assume

$$
\int_{\mathbf{D}} g\left(\bar{w} \phi_{w}(z)\right) \frac{\varphi(w)}{(1-\bar{w} z)^{2}} d A(w)=\varphi(z)
$$

for all $\varphi \in A^{2}$. Given $\psi \in A^{2}$ and $z \in D$, consider $\varphi(w)=\psi\left(\phi_{z}(w)\right) \frac{\left(1-|z|^{2}\right)^{2}}{(1-\bar{z} w)^{2}}$. Clearly $\varphi \in A_{2}$ and $\|\varphi\|_{2}=\left(1-|z|^{2}\right)\|\psi\|_{2}$. From the assumption,

$$
\int_{\mathbf{D}} g\left(\bar{w} \phi_{w}(z)\right) \psi\left(\phi_{z}(w)\right) \frac{\left(1-|z|^{2}\right)^{2}}{|1-\bar{w} z|^{4}} d A(w)=\psi(0) .
$$

for all $\psi \in A^{2}$ and $z \in \mathbf{D}$.

Now changing the variable $u=\phi_{z}(w)$, and using (6), one gets

$$
\int_{\mathbf{D}} g\left(u \overline{\phi_{u}(z)}\right) \psi(u) d A(u)=\psi(0)
$$

for all $\psi \in A_{2}$ and $z \in \mathbf{D}$. Finally changing $u$ by $\bar{w}$ one obtains

$$
\int_{\mathbf{D}} g\left(\bar{w} \phi_{w}(z)\right) \psi(\bar{w}) d A(w)=\psi(0)
$$

for all $\psi \in A_{2}$ and $z \in \mathbf{D}$. (i) follows selecting $\psi=1$ and $z=0$ in (10). 
Differentiating in $(10)$ with respect to $z$ one obtains

$$
\int_{\mathbf{D}} g^{\prime}\left(\bar{w} \phi_{w}(z)\right) \frac{-\bar{w}\left(1-|w|^{2}\right)}{(1-\bar{w} z)^{2}} \psi(\bar{w}) d A(w)=T_{g^{\prime}}\left(\psi_{1}\right)=0
$$

where $\varphi_{1}(u)=-\bar{u}\left(1-|u|^{2}\right) \varphi(\bar{u})$. Hence (ii) is finished.

Let us now show that $\left(\mathscr{P},\|\cdot\|_{\mathscr{P}}\right)$ is complete. For such a purpose, let us define $h_{z}: \mathbf{D} \rightarrow \mathbf{H}$ by

and let us mention that

$$
h_{z}(w)=\frac{1}{\psi_{z}(w)}=\frac{1-z \bar{w}}{1-|w|^{2}}
$$

$$
\mathbf{D}_{1}=\left\{\frac{1-|w|^{2}}{1-z \bar{w}}: z, w \in \mathbf{D}\right\}=\left\{\psi_{z}(w): z, w \in \mathbf{D}\right\}
$$

Lemma 12. For every $\xi \in \mathbf{H}$, there exist $0 \leq \alpha<1$ and $w \in \mathbf{D}$ such that $\xi=h_{\alpha}(w)$ and $h_{\alpha}$ is an diffeomorfism of a neighborhood $U$ of $w$ onto an open neighborhood of $\xi$.

Proof. For $0 \leq r, \alpha<1$ fixed,

$$
h_{\alpha}\left(r e^{i \theta}\right)=\frac{1}{1-r^{2}}-\frac{r \alpha}{1-r^{2}} e^{-i \theta}
$$

describes the circle $C_{r, \alpha}$ centered at the complex number $\frac{1}{1-r^{2}}$ with radius $\frac{r \alpha}{1-r^{2}}$. Let $\xi \in \mathbf{H}$. To prove that $\xi \in h_{\alpha}(\mathbf{D})$ it is enough to see that $\xi \in C_{r, \alpha}$ for some $0 \leq r, \alpha<$ 1. Let

$$
\beta=\frac{1}{r^{2}}\left[\left(1-r^{2}\right)^{2}|\xi|^{2}+1-2\left(1-r^{2}\right) \operatorname{Re} \xi\right]=\frac{\left|\left(1-r^{2}\right) \xi-1\right|^{2}}{r^{2}} .
$$

It is clear that $\beta \geq 0$ and

$$
\beta<1 \Leftrightarrow\left(1-r^{2}\right)|\xi|^{2}+1<2 \operatorname{Re} \xi
$$

Also, since $\xi \in \mathbf{H}$, we have for some $\varepsilon>0$ that $2 \operatorname{Re} \xi>1+\varepsilon$. Hence if $|\xi|^{2}<\frac{\varepsilon}{\left(1-r^{2}\right)}$ then $\beta<1$. We conclude that there exists $r_{0}$ for which $0 \leq \beta<1$ provided $r_{0}<r<1$. Then if $r_{0}<r<1$ and $\alpha=\sqrt{\beta}$ we have $0 \leq \alpha<1$ and

$$
\left|\xi-\frac{1}{1-r^{2}}\right|=\frac{r \alpha}{1-r^{2}},
$$

that is $\xi \in C_{r, \alpha}$. Hence there exists $\theta_{r}$ and $0 \leq \alpha_{r}<1$ such that $h_{\alpha_{r}}\left(r e^{i \theta_{r}}\right)=\xi$.

To find $\theta_{r}$ explicitly, we let $\varphi_{r}=\pi-\theta_{r}$. From (11) we can write

$$
\xi=\frac{1}{1-r^{2}}+\frac{r \alpha_{r}}{1-r^{2}} e^{i \varphi_{r}}
$$

Hence $\varphi_{r}$ is the argument of $\xi$ in polar coordinates centered at the complex number $\frac{1}{1-r^{2}}$. Then if $\frac{1}{1-r^{2}} \geq \operatorname{Re}(\xi)$,

$$
\begin{aligned}
& \sin \theta_{r}=\sin \varphi_{r}=\frac{\operatorname{Im}(\xi)}{r \alpha_{r}}\left(1-r^{2}\right) \\
& \cos \theta_{r}=-\cos \varphi_{r}=\frac{\left(1-r^{2}\right)}{r \alpha_{r}}\left(\frac{1}{1-r^{2}}-\operatorname{Re}(\xi)\right)=\frac{1-\left(1-r^{2}\right) \operatorname{Re}(\xi)}{r \alpha_{r}} .
\end{aligned}
$$

Now we will prove that possibly except for a finite number of values of $r \geq r_{0}$, the jacobian matrix $D h_{\alpha_{r}}\left(r e^{i \theta_{r}}\right)$ is not singular, where $\alpha_{r}$ and $\theta_{r}$ are chosen so that 
$h_{\alpha_{r}}\left(r e^{i \theta_{r}}\right)=\xi$ as before. To this end, it is enough to see that the set of values of $r$ for which the vectors

$$
\frac{\partial h_{a_{r}}}{\partial \rho}\left(\rho e^{i \theta_{r}}\right)_{\mid \rho=r} \text { and } \frac{1}{r} \frac{\partial h_{a_{r}}}{\partial \theta}\left(r e^{i \theta}\right)_{\mid \theta=\theta_{r}}
$$

are linearly dependent is finite.

We have

$$
\begin{aligned}
\frac{\partial h_{a}}{\partial \rho}\left(\rho e^{i \theta}\right) & =\left(\frac{2 \rho}{\left(1-\rho^{2}\right)^{2}}-\frac{\alpha\left(1+\rho^{2}\right)}{\left(1-\rho^{2}\right)^{2}} \cos \theta, \frac{\alpha\left(1+\rho^{2}\right)}{\left(1-\rho^{2}\right)^{2}} \sin \theta\right), \\
\frac{1}{\rho} \frac{\partial h_{a}}{\partial \theta}\left(\rho e^{i \theta}\right) & =\left(\frac{\alpha}{\left(1-\rho^{2}\right)} \sin \theta, \frac{\alpha}{\left(1-\rho^{2}\right)} \cos \theta\right),
\end{aligned}
$$

and the jacobian of $h_{\alpha}$

$$
\begin{aligned}
J h_{\alpha}\left(\rho e^{i \theta}\right) & =\operatorname{det}\left[\frac{\partial h_{a}}{\partial \rho}\left(\rho e^{i \theta}\right) \mid \frac{1}{\rho} \frac{\partial h_{a}}{\partial \theta}\left(\rho e^{i \theta}\right)\right] \\
& =\operatorname{det}\left[\begin{array}{cc}
\frac{2 \rho}{\left(1-\rho^{2}\right)^{2}}-\frac{\alpha\left(1+\rho^{2}\right)}{\left(1-\rho^{2}\right)^{2}} \cos \theta & \frac{\alpha\left(1+\rho^{2}\right)}{\left(1-\rho^{2}\right)^{2}} \sin \theta \\
\frac{\alpha}{\left(1-\rho^{2}\right)} \sin \theta & \frac{\alpha}{\left(1-\rho^{2}\right)} \cos \theta
\end{array}\right] \\
& =\frac{\alpha}{\left(1-\rho^{2}\right)^{3}}\left(2 \rho \cos \theta-\alpha\left(1+\rho^{2}\right)\right) .
\end{aligned}
$$

If $2 r \cos \theta_{r}-\alpha_{r}\left(1+r^{2}\right)=0$, then multiplying this equation by $\alpha_{r} r^{2}$ we obtain

$$
2 r^{2} \alpha_{r} r \cos \theta_{r}-\alpha_{r}^{2} r^{2}\left(1+r^{2}\right)=0 .
$$

However, from (12) and (13) we see that $2 r^{2} \alpha_{r} r \cos \theta_{r}-\alpha_{r}^{2} r^{2}\left(1+r^{2}\right)$ is a polynomial of degree 6 in the variable $r$. We conclude that the vectors in (14) are linearly dependent for six values of $r$ at the most and the proof of the lemma is complete.

Theorem 13. $\mathscr{P}$ is a Banach space.

Proof. Let $g \in \mathscr{P}$. We have by Theorem 8 that

$$
\sup _{z \in \mathbf{D}}\left\{\int_{\mathbf{D}}\left|g\left(\bar{w} \phi_{w}(z)\right)\right|^{2} d A(w)\right\}^{1 / 2} \leq 2\|g\|_{\mathscr{P}} .
$$

Fix $\xi \in \mathbf{D}$. Since $\psi_{z}=1 / h_{z}$, the local invertibility statement of Lemma 12 holds for the family of functions $1-\psi_{z}$ taking $\xi \in \mathbf{D}$, namely, there exist $\alpha \in(0,1), w_{\xi} \in \mathbf{D}$ and open neighborhoods $U$ and $V$ of $\xi$ and $w_{\xi}$ respectively, such that $1-\psi_{z}$ is a diffeomorphism of $V$ into $U$.

Hence

$$
\begin{aligned}
\left\{\int_{U}|g(u)|^{2} d A(u)\right\}^{1 / 2} & =\left\{\int_{V}\left|g\left(1-\psi_{\alpha}(w)\right)\right|^{2}\left|J \psi_{\alpha}(w)\right| d A(w)\right\}^{1 / 2} \\
& \leq C(\xi)\left\{\int_{V}\left|g\left(\bar{w} \phi_{w}(\alpha)\right)\right|^{2} d A(w)\right\}^{1 / 2} \\
& \leq C(\xi)\|g\|_{\mathscr{P}} .
\end{aligned}
$$


It follows that

$$
\left\{\int_{K}|g(u)|^{2} d A(u)\right\}^{1 / 2} \leq C_{K}\|g\|_{\mathscr{P}},
$$

for every compact set $K \subset \mathbf{D}$. This implies that

$$
\sup _{u \in K}|g(u)| \leq\|g\|_{\mathscr{P}} C_{K}^{\prime}
$$

If $\left\{g_{n}\right\}$ is a Cauchy sequence in $\mathscr{P}$, we have by (18) that $\left\{g_{n}\right\}$ converges uniformly on compact sets of $\mathbf{D}$ to a holomorphic function $g$.

Let us show that $g \in \mathscr{P}$ and $\left\|g_{n}-g\right\|_{\mathscr{P}} \rightarrow 0$. Note first that for each $\varphi \in C_{c}(\mathbf{D})$ we have

$$
T_{g_{n}} \varphi(z) \rightarrow T_{g} \varphi(z), \quad z \in \mathbf{D} .
$$

Using the fact $\sup _{n \in \mathbf{N}}\left\|g_{n}\right\|_{\mathscr{P}}=M<\infty$ and Fatou's lemma one gets

$$
\left\|T_{g} \varphi\right\|_{2}^{2} \leq \liminf _{n \rightarrow \infty}\left\|T_{g_{n}} \varphi\right\|_{2}^{2} \leq M\|\varphi\|_{2}^{2} .
$$

Hence $g \in \mathscr{P}$. On the other hand, given $\varepsilon>0$ there exists $n_{0}$ such that

$$
\left\|T_{g_{n}} \varphi-T_{g_{m}} \varphi\right\|_{2} \leq\left\|g_{n}-g_{m}\right\|_{\mathscr{P}}<\varepsilon
$$

for $m, n \geq n_{0}$ and $\|\varphi\|_{2}=1$. Applying Fatou's lemma again we conclude that

$$
\left\|T_{g_{n}} \varphi-T_{g} \varphi\right\|_{2} \leq \varepsilon
$$

for $n \geq n_{0}$. Therefore $g_{n} \rightarrow g$ in $\mathscr{P}$.

\section{Main results}

Let us now describe the norm in $\mathscr{P}$ in a more explicit way. We shall use the formulation of the space given in [1].

Theorem 14. Let $g \in \mathscr{H}(\mathbf{D})$ and put $F(\xi)=\frac{1}{\xi^{2}} g\left(1-\frac{1}{\xi}\right)$. Then $g \in \mathscr{P}$ if and only if

$$
\sup _{j} \frac{1}{j ! \sqrt{j+1}}\left(\int_{1}^{\infty}[(x-1) x]^{j}\left|x F^{(j)}(x)\right|^{2} d x\right)^{1 / 2}<\infty .
$$

Proof. We use the expression

$$
T_{g} \varphi(z)=\int_{\mathbf{D}} F\left(\frac{1-z \bar{w}}{1-|w|^{2}}\right) \varphi(w) \frac{d A(w)}{\left(1-|w|^{2}\right)^{2}} .
$$

Consider the space $M$ of functions of the form

$$
\varphi=\sum_{\text {finite }} \varphi_{j}(r) e^{i j \theta}
$$

with $\varphi_{j} \in L^{2}((0,1), r d r)$. Then $M$ is a dense subspace of $L^{2}(\mathbf{D})$.

For $z \in \mathbf{D}$ and $0 \leq r<1$ fixed, let $f(\zeta)=F\left(\frac{1-r z \zeta}{1-r^{2}}\right)$, which is holomorphic on $\overline{\mathrm{D}}$. We have

$$
f(\zeta)=F\left(\frac{1-r z \zeta}{1-r^{2}}\right)=\sum_{j \geq 0} \frac{1}{j !}\left(\frac{-r z}{1-r^{2}}\right)^{j} F^{(j)}\left(\frac{1}{1-r^{2}}\right) \zeta^{j}, \quad|\zeta| \leq 1 .
$$


Then for $g \in M$,

$$
\int_{0}^{2 \pi} f\left(r e^{-i \theta}\right) \varphi\left(r e^{i \theta}\right) \frac{d \theta}{2 \pi}=\sum_{j \geq 0} \varphi_{j}(r) \frac{(-1)^{j}}{j !}\left(\frac{r}{1-r^{2}}\right)^{j} F^{(j)}\left(\frac{1}{1-r^{2}}\right) z^{j},
$$

Hence

$$
T_{g}(\varphi)(z)=\sum_{j \geq 0} \gamma_{j}\left(\varphi_{j}\right) \sqrt{j+1} z^{j}
$$

where $\gamma_{j}$ is the functional in $L^{2}((0,1), r d r)$ defined by

$$
\gamma_{j}(\varphi)=\frac{(-1)^{j}}{\sqrt{j+1} j !} \int_{0}^{1} \varphi(r)\left(\frac{r}{1-r^{2}}\right)^{j} F^{(j)}\left(\frac{1}{1-r^{2}}\right) \frac{r}{\left(1-r^{2}\right)^{2}} d r .
$$

Using the normalized Lebesgue measure $d A$, the set $\left\{\sqrt{j+1} z^{j}\right\}$ is an orthonormal basis for $A^{2}$, so we conclude that $T_{g}$ is bounded in $L^{2}(\mathbf{D})$ if and only if

$$
\left\|\left(\gamma_{j}\left(\varphi_{j}\right)\right)_{j \geq 0}\right\|_{\ell^{2}} \leq C\|\varphi\|_{L^{2}(\mathbf{D})}=C\left(\sum_{j} \int\left|\varphi_{j}(r)\right|^{2} r d r\right)^{1 / 2} .
$$

Using duality, this will hold if and only if

$$
\sup _{j \geq 0} \frac{1}{\sqrt{j+1} j !}\left(\int_{0}^{1}\left(\frac{r}{1-r^{2}}\right)^{2 j}\left|F^{(j)}\left(\frac{1}{1-r^{2}}\right)\right|^{2} \frac{r d r}{\left(1-r^{2}\right)^{4}}\right)^{1 / 2}<\infty .
$$

Making the change of variables $x=\frac{1}{1-r^{2}}$, the integrals above equal

$$
\frac{1}{2} \int_{1}^{\infty}[(x-1) x]^{j}\left|x F^{(j)}(x)\right|^{2} d x
$$

and the proof is complete.

We can now give an alternative proof of a well know result.

Corollary 15. $P_{\alpha}$ is bounded on $L^{2}(\mathbf{D})$ if and only if $\alpha>-1 / 2$.

Proof. Consider $g_{\alpha}(z)=(1-z)^{\alpha}$. Assume first that $g_{\alpha} \in \mathscr{P}$. Then (3) in Theorem 8 implies that $\int_{0}^{1}(1-r)^{2 \alpha} d r<\infty$ and therefore $\alpha>-1 / 2$.

Assume now that $\alpha>-1 / 2$. Since $F_{\alpha}(\xi)=\xi^{-m}$ with $m=2+\alpha$ and $2 m-3>0$, one has for $j \geq 0$ that

$$
F_{\alpha}^{(j)}(x)=(-1)^{j} m(m+1) \cdots(m+j-1) x^{-(m+j)}=(-1)^{j} \frac{\Gamma(m+j)}{\Gamma(m)} x^{-(m+j)} .
$$

Therefore

$$
\begin{aligned}
\int_{1}^{\infty}[(x-1) x]^{j}\left|x F_{\alpha}^{(j)}(x)\right|^{2} d x & =\int_{1}^{\infty}\left(1-\frac{1}{x}\right)^{j}\left(x^{j+1} F_{\alpha}^{(j)}(x)\right)^{2} d x \\
& =\left(\frac{\Gamma(m+j)}{\Gamma(m)}\right)^{2} \int_{1}^{\infty}\left(1-\frac{1}{x}\right)^{j} x^{-2 m+4} \frac{d}{x^{2}} \\
& =\left(\frac{\Gamma(m+j)}{\Gamma(m)}\right)^{2} \int_{0}^{1}(1-r)^{j} r^{2 m-4} d r \\
& =\left(\frac{\Gamma(m+j)}{\Gamma(m)}\right)^{2} B(2 m-3, j+1)
\end{aligned}
$$


Using $B(p, q)=\frac{\Gamma(p) \Gamma(q)}{\Gamma(p+q)}$ one concludes that

$$
\frac{1}{(j !)^{2}(j+1)} \int_{1}^{\infty}[(x-1) x]^{j}\left|x F_{\alpha}^{(j)}(x)\right|^{2} d x=\frac{B(2 m-3, j+1)}{B(m, j)^{2} j^{2}(j+1)} .
$$

Finally since for $p$ fixed, $B(p, j) \sim j^{-p}$ one obtains

$$
\frac{B(2 m-3, j+1)}{B(m, j)^{2} j^{2}(j+1)} \sim 1
$$

Example 16. In Example 7 it was shown that, for $0<\beta<5 / 4, g(z)=(1+$ $z)^{-\beta} \in \mathscr{P}$ (which corresponds to $F(\xi)=\frac{\xi^{\beta-2}}{(2 \xi-1)^{2}}$ ). Let us show, for instance, that $g(z)=(1+z)^{-2} \notin \mathscr{P}$. In this case $F(\xi)=\frac{1}{(2 \xi-1)^{2}}$ and

$$
F^{(j)}(\xi)=\frac{(-1)^{j}(j+1) ! 2^{j}}{(2 \xi-1)^{2+j}}
$$

Since $\frac{x}{2} \leq x-1 \leq x$ for $x \geq 2$ we have

$$
\left(\int_{2}^{\infty}(x(x-1))^{j}\left|x F^{(j)}(x)\right|^{2} d x\right)^{1 / 2} \sim 2^{j}(j+1) !\left(\int_{2}^{\infty} \frac{x^{2 j+2}}{(2 x-1)^{4+2 j}} d x\right)^{1 / 2} \sim 2^{j}(j+1) ! .
$$

Hence the condition in Theorem 14 does not hold.

The conditions

$$
\begin{array}{r}
\sup _{j \geq 0} \frac{1}{j !} \int_{1}^{\infty}\left|(x-1)^{j} F^{(j)}(x)\right| d x<\infty \\
\lim _{x \rightarrow \infty} x^{j+1} F^{(j)}(x)=0
\end{array}
$$

were introduced in [1]. These conditions imply that on the space of all the holomorphic functions $\varphi$ such that $S_{F} \varphi$ is well defined, the operator $S_{F}$ is a constant multiple of the identity. Now we will see that (21) and (22) hold for every $g \in \mathscr{P}$ which allows to show the following result.

Theorem 17. Let $g \in \mathscr{P}$ and $c_{0}=\int_{0}^{1} g(r) d r$. Then

$$
T_{g}(\varphi)=c_{0} \varphi, \quad \varphi \in A^{2} .
$$

Proof. Let us notice first that $(x-1)^{j} F^{(j)}(x) \in L^{1}([1, \infty), d x)$ for $j \geq 0$. Indeed,

$$
\begin{aligned}
& \int_{1}^{\infty}|x-1|^{j}\left|F^{(j)}(x)\right| d x=\int_{1}^{\infty}|x(x-1)|^{j}\left|x F^{(j)}(x)\right| \frac{d x}{x^{j+1}} \\
& \leq\left(\int_{1}^{\infty}(x(x-1))^{j}\left|x F^{(j)}(x)\right|^{2} d x\right)^{1 / 2}\left(\int_{1}^{\infty} \frac{(x(x-1))^{j}}{x^{2 j+2}} d x\right)^{1 / 2} \\
& =\left(\int_{1}^{\infty}(x(x-1))^{j}\left|x F^{(j)}(x)\right|^{2} d x\right)^{1 / 2}\left(\int_{0}^{1}(1-r)^{j} d r\right)^{1 / 2} \\
& =\frac{1}{\sqrt{j+1}}\left(\int_{1}^{\infty}|x(x-1)|^{j}\left|x F^{(j)}(x)\right|^{2} d x\right)^{1 / 2} \leq C j !\|g\|_{\mathscr{P} .}
\end{aligned}
$$

Applying (19) in Theorem 14 to $\varphi(z)=\sum_{j=0}^{N} a_{j} z^{j}$ one obtains

$$
T_{g} \varphi=\sum_{j=0}^{N} c_{j} a_{j} z^{j},
$$


and

$$
c_{j}=\frac{(-1)^{j}}{j !} \int_{1}^{\infty}(x-1)^{j} F^{(j)}(x) d x
$$

where $c_{j}$ is well defined. As in [1, Th. 1] we have by integration by parts

$$
c_{j}-c_{j+1}=\frac{(-1)^{j}}{(j+1) !} \lim _{x \rightarrow \infty}(1-x)^{j+1} F^{(j)}(x) .
$$

Let us now show that $\lim _{x \rightarrow \infty}(1-x)^{j+1} F^{(j)}(x)=0$. Note first that $(x-$ $1)^{j+1} F^{(j)}(x) \in L^{2}([1, \infty), d x)$ for $j \geq 0$. Indeed

$$
\int_{1}^{\infty}\left|(x-1)^{j+1} F^{(j)}(x)\right|^{2} d x \leq \int_{1}^{\infty}|x(x-1)|^{j}\left|x F^{(j)}(x)\right|^{2} d x \leq C(j+1)(j !)^{2} .
$$

In particular $(x-1)^{j} F^{(j)}(x) \in L^{2}([1, \infty), d x)$ for $j \geq 1$. From Cauchy-Schwarz and the previous estimates one has that if $f_{j}(x)=\left[(x-1)^{j+1} F^{(j)}(x)\right]^{2}$, then $\left(f_{j}\right)^{\prime} \in$ $L^{1}([1, \infty))$ for every $j \geq 0$. Therefore writing

$$
\left[(x-1)^{j+1} F^{(j)}(x)\right]^{2}=\int_{1}^{x}\left(f_{j}\right)^{\prime}(y) d y
$$

we see that the $\lim _{x \rightarrow \infty}\left((x-1)^{j+1} F^{(j)}(x)\right)^{2}$ exists and by (24) it vanishes for all $j$. Hence (23) becomes $T_{g}(\varphi)=c_{0} \varphi$ where

$$
c_{0}=\int_{1}^{\infty} F(x) d x=\int_{1}^{\infty} g\left(1-\frac{1}{x}\right) \frac{d x}{x^{2}}=\int_{0}^{1} g(r) d r .
$$

Corollary 18. Let $g \in \mathscr{P}$. Then $A^{2} \subset \operatorname{Ker} T_{g}$ if and only if $\int_{0}^{1} g(r) d r=0$.

Corollary 19. Let $\Phi(g)=\int_{0}^{1} g(r) d r$ for $g \in \mathscr{P}$. Then $\mathscr{P}_{0}=\Phi^{-1}(\{1\})$.

Corollary 20. Let $g \in \mathscr{P}$. If $T_{g}$ is not identically zero in $A^{2}$ then there exists $\lambda \neq 0$ and $g_{0} \in \mathscr{P}_{0}$ such that $g=\lambda g_{0}$.

\section{References}

[1] Bonet, J., M. Engliš, and J. TAskinen: Weighted $L^{\infty}$-estimates for Bergman projections. Studia Math. 171:1, 2005, 67-92.

[2] Dostanić, M.: Norm estimate of the Cauchy transform on $L^{p}(\Omega)$. - Integral Equations Operator Theory 52:4, 2005, 465-475.

[3] Dostanić, M.: Norm of Berezin transform on $L^{p}$ space. - J. Anal. Math. 104:1, 2008, 13-23.

[4] Forelli, F., and W. Rudin: Projections on spaces of holomorphic functions on balls. - Indiana Univ. Math. J. 24, 1974, 593-602.

[5] Hedenmalm, H., B. Korenblum, and K. Zhu: Theory of Bergman spaces. - Grad. Texts in Math. 199, Springer-Verlag, 2000.

[6] Rudin, W.: Function theory in the unit ball of $\mathbf{C}^{n}$. - Springer, New York, 1980.

[7] ZHU, K.: Operator theory in function spaces. - Marcel Dekker, New York, 1990. 